\title{
Impressum, Vol. 3, No. 4, 1980
}

\section{Herausgeber}

Prof. Dr. S. Eckhardt, Staatliches Onkologisches Institut, Budapest

Prof. Dr. R. Gross, Medizinische Universitätsklinik, Köln

Prof. Dr. J. H. Holzner, Institut für pathologische Anatomie,

Universität Wien

Prof. Dr. K. Munk, Deutsches Krebsforschungszentrum, Institut für

Virusforschung, Heidelberg

Prof. Dr. G. P. Murphy, Roswell Park Memorial Institute,

Buffalo (N.Y.)

Prof. Dr. A. Stacher, Hanusch-Krankenhaus, Wien

Prof. Dr. Dr. St. Tanneberger, Zentralinstitut für Krebsforschung,

Akademie der Wissenschaften, Berlin/DDR

Prof. Dr. W. Wilmanns, Medizinische Klinik III, Klinikum Groß-

hadern, Universität München

Prof. Dr. Dr. H. Wrba, Institut für Krebsforschung, Universität Wien

Wissenschaftlicher Beirat

H. W. Bauer, München; H. Denck, Wien; V. Diehl, Hannover; F. Douwes, Göttingen; P. Drings, Heidelberg; H. Ehrhart, München; J. Fischer, Mainz; W. M. Gallmeier, Nürnberg; H. Gerhartz, Berlin; E. H. Graul, Marburg/Lahn; E. Hauptmann, Zagreb; H. Heimpel, Ulm; K. P. Hellriegel, Köln; Ch. Herfarth, Ulm; R. Hünig, Basel; W. Hunstein, Heidelberg; H. O. Klein, Köln; H.

Löffler, Lahn Gießen; U. Mohr, Hannover; F.-L. Müller, Bonn; G. Nagel, Göttingen; A.

Pfleiderer, Freiburg; Mildred Scheel, Bonn; E. Scherer, Essen; S. Seeber, Essen; H. J. Senn, St.

Gallen; W. Vahlensieck, Bonn; H. D. Waller, Tubingen; M. Wannenmacher, Freiburg; K.

Wilms, Tubingen; K. zum Winkel, Heidelberg

Offizíelles Organ der Deutschen Gesellschaft für Hämatologie und Onkologie

und der österreichischen Gesellschaft für Hämatologie und Onkologie

Satz und Druck, Walter Biering GmbH, Grafischer Betrieb, 8000 München 45.

Für den Inhalt außerhalb des redaktionellen Teiles (insbesondere Anzeigen,

Industrieinformationen, Pressezitate und Kongreßinfor-mationen) übernehmen Herausgeber und Verlag keine Gewähr.

Alle Rechte, insbesondere das Recht der Vervielfältigung und Mikro-kopie sowie der

Übersetzung, vorbehalten. Nachdruck, auch auszugs-weise, nur mit Genehmigung des Verlages.

(C) Copyright 1980 by S. Karger Verlag für Medizin und Naturwissen-schaften GmbH, Postfach

2, D-8034 Germering

Die Zeitschrift erscheint zweimonatlich; pro Jahr erscheint 1 Band zu je 6 Heften.

Bezugspreis für Jahrgang 3, 1980 DM 104,-/OS 728,-/SFr. 104,-. 1 Einzelheft kostet DM 22,-

/OS 154,-/SFr. 22,-, einschließlich MwSt., zuzüglich Postgebühren.

Der Abonnementspreis ist im voraus zahlbar. Die Lieferung der Zeitschrift läuft weiter, wenn sie nicht spätestens 4 Wochen vor Abschluß eines Bandes abbestellt wird. 
Abonnementsbestellungen können bei jeder Buchhandlung, bei der Post oder direkt beim Verlag aufgegeben werden: Bundesrepublik Deutschland/Österreich: S. Karger GmbH, Postfach 2, D8034 Germering/München, Tel: (089) 844021, Telex: 524865 D, Postscheckkonto: München 40080-807 Schweiz: S. Karger AG, AПschwilerstr. 10, Postfach, CH-4009 Basel, Tel.: (061) 3908 80, Telex $62652 \mathrm{CH}$

\section{Anzeigen}

S. Karger Verlag für Medizin und Naturwissenschaften GmbH, Postfach 2, 8034 Germering, Telefon (089) 844021.

Sonderhefte bzw. Sonderbände sind nur in beschränkter Auflage vor-rätig. Der Preis ist abhängig vom Umfang. Abonnenten erhalten diese zu einem um 20 \% vom Ladenpreis ermäßigten Vorzugspreis.

Beiträge zur Onkologie

Sonderband 1: Chemotherapie urologischer Malignome

Prostata, Harnblase, Penis/Harnröhre. Seminar des Berufsverbandes

Deutscher Urologen e. V., Mainz, März 1979

190 S., 70 Abb., 59 Tab., brosch., DM 35-

Sonderband 2: Chirurgische Therapie des Mammakarzinoms.

1. Österreichische Chirurgentagung, Salzburg, September 1978.

VIII, 60 S., 16 Abb., brosch., DM 25-

Sonderband 3: CISPLATIN. Derzeitiger Stand und neue Entwick-

Iungen in der Chemotherapie maligner Neoplasien.

Symposium am 16. November 1979, Frankfurt a. M.

VIII, 176 S., 37 Abb., 89 Tab., brosch., DM 35,-

Sonderband 4: Probleme der Krebsnachsorge. Prognose, Begutachtung und Rehabilitation bei gynäkologischen Karzinomen.

VIII, 140S., 32 Abb., 13 Tab., brosch., DM40-

Sonderband 5: Neue Erfahrungen mit Oxazaphosphorinen unter besonderer Berücksichtigung des Uroprotektors Uromitexan ${ }^{\circledR}$.

Gemeinsames Symposium der Arbeitsgemeinschaft für internistische

Onkologie (AIO) der Deutschen Krebsgesellschaft und der ASTA-

Werke AG, Bielefeld, am 29. Februar 1980 in Düsseldorf.

X, 126 S., 36 Abb., 49 Tab., brosch., DM 35-

Beiträge zur Urologie

Sonderband 1: Diagnostik und Therapie des Prostatakarzinoms Symposium der Urologischen Universitätsklinik München in Zusam-menarbeit mit dem Tumorzentrum München, München, November '78 168 S., 67 Abb., 48 Tab., brosch., DM 40- 